\title{
Fouiller les mots
}

\section{Rudolf Wachter}

\section{(2) OpenEdition}

Journals

Édition électronique

URL : http://journals.openedition.org/edl/406

DOI : $10.4000 /$ edl. 406

ISSN : 2296-5084

\section{Éditeur}

Université de Lausanne

\section{Édition imprimée}

Date de publication : 15 mai 2010

Pagination : 275-292

ISBN : 978-2-940331-22-2

ISSN : 0014-2026

\section{Référence électronique}

Rudolf Wachter, «Fouiller les mots», Études de lettres [En ligne], 1-2 | 2010, mis en ligne le 15 mai 2013, consulté le 21 décembre 2020. URL : http://journals.openedition.org/edl/406 ; DOI : https:// doi.org/10.4000/edl.406 


\section{FOUILLER LES MOTS}

Tout comme le terme archaiología et le verbe correspondant qui se rapportaient à la «discussion de sujets traditionnels" chez les Grecs de l'Antiquité, des «fouilles archéologiques" ne sont pas seulement possibles dans le sol que nous avons repris de nos ancêtres; de manière similaire, notre langue est riche en objets de famille qui nous ont été légués par ces derniers et qu'il vaut la peine de "fouiller». Car notre langue est le miroir le plus clair de notre culture et de sa genèse. Il s'agit alors de chercher derrière les mots les "étymologies", les "vraies explications", qui sont souvent bien cachées. Elles se trouvent dans les couches anciennes des vocables et du système linguistique, en grande partie héritées des profondeurs du temps, mais recouvertes par des changements plus ou moins récents. Le meilleur moyen de les déterrer est fourni par la méthode de la comparaison linguistique historique. Le lecteur est invité à prendre part à une série de fouilles de mots fascinantes, entre autres pain, lait, cas, tu iras et inspiration.

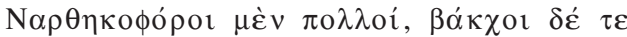
$\pi \alpha \tilde{\text { pot. }}$

Platon, Phédon 69c

Le sujet de ce colloque, la tradition classique, implique un engagement énorme du côté des savants et de leur public. Les archéologues nous emmènent dans des sites d'habitat, des sanctuaires antiques ou dans des musées où les objets trouvés sont présentés et expliqués. Ils nous recommandent la lecture de livres bien illustrés, avec descriptions et interprétations des trouvailles. Les philologues nous parlent des œuvres écrites de l'Antiquité et de leur influence sur l'art et la littérature modernes, les films d'Hollywood inclus. Ils essaient de mettre en contact leurs contemporains avec les fondements de la pensée occidentale. Les historiens reconstruisent la politique des époques passées en mettant tout en rapport: la société et l'individu, les religions, les coutumes, la culture, 
les sources. Et ils espèrent que nous, le public, serons prêts à dépenser de l'énergie, du temps et de l'argent pour mieux connaître cette tradition.

Bien sûr, le film Troy ne touche pas les jeunes d'aujourd'hui parce que l'histoire qu'il raconte remonte à 2700 ans avant nos jours, mais parce qu'elle les fait réfléchir sur l'absurdité de la guerre, sur la proximité de la vie et de la mort. Une minorité, peut-être, y cherche une réponse à la question homérique: vérité historique ou fiction? De même, La Peste de Camus avec ses sources antiques, à savoir les passages sur la peste à Athènes chez Thucydide et chez Lucrèce, nous touche parce qu'elle nous montre, d'une manière choquante, l'absurdité de la maladie, symbole de la guerre, et l'impuissance totale de l'être humain vis-à-vis de la mort ${ }^{1}$. La littérature antique nous offre des cas exemplaires de la condition humaine, des exemples qui, malgré - ou précisément à cause de? - leur distance temporelle, nous touchent par leur proximité culturelle, et surtout morale. L'archéologie, de son côté, nous frappe par les contrastes qu'elle fait ressortir: c'est le mélange entre l'existence dans l'Antiquité d'objets dont nous ne pensions peut-être pas qu'ils existaient déjà à une époque aussi reculée, et l'absence totale d'objets sans lesquels nous ne pouvons presque plus nous imaginer vivre. Là aussi, c'est le sentiment d'une proximité, par exemple l'identité du terrain que nous habitons et qu'ont habité les gens dans l'Antiquité, et en même temps le sentiment d'une distance énorme qui nous touchent.

Néanmoins, ces parallèles antiques nous sont a priori étrangers. Les textes antiques ne sont normalement pas des éléments constitutifs de notre vie de tous les jours, et les vestiges archéologiques également ne nous sont pas automatiquement proches, même aux habitants d'une ville comme Rome où, au moins au centre, il y a des traces de l'Antiquité partout. En tant que chercheurs et amateurs de la tradition classique, nous savons bien quels efforts il faut fournir pour rendre proches à nos contemporains les valeurs de nos domaines.

Outre le terrain et les objets concrets de la vie humaine d'un côté et la pensée et l'interaction sociale et politique de l'autre, il est un troisième domaine dont la profondeur historique est fascinante à découvrir: la langue. En fait, elle fournit un rapport encore plus direct et étroit entre nous-mêmes et le passé. Car il ne nous faut ni nous déplacer, ni ouvrir un livre, ni même ouvrir nos yeux; nous avons en principe tout

I. Voir les contributions de D. Bouvier et de D. van Mal-Maeder dans ce volume. 
ce qu'il faut en nous-mêmes, dans nos cerveaux. Mais là aussi, la profondeur historique ne se révèle pas automatiquement. Moyen culturel le plus important mais en même temps le plus naturel et inévitable ${ }^{2}$, commun à pratiquement tous les êtres humains de plus de deux ans, la langue n'est pour la plupart des gens qu'un outil et guère un objet de réflexion. Quand nous en devenons conscients, pour une ou deux secondes, c'est parce que quelqu'un a commis une faute ou nous a corrigés. Mais la conscience excède rarement le désir de savoir ce qui est correct et ce qui ne l'est pas - ce que j'appelle la question de l'élève, la question du «comment?». Une telle restriction est regrettable, car ce n'est qu'avec la question "pourquoi telle ou telle expression est-elle fausse et telle autre correcte?» que la réflexion sur la langue devient vraiment intéressante. Voilà donc la question du chercheur, du philosophe qui cherche les causes en demandant "pourquoi?». A la fouille du terrain et à celle des textes s'associe donc un troisième type de fouille, celle de la langue.

Déjà synchroniquement, il vaut la peine de réfléchir sur la langue. Cet instrument de communication est l'un des objets les plus complexes qui existe en nous, et le plus proche de notre identité qui nous soit encore accessible consciemment, tandis que le corps et l'âme et leurs communications non verbales échappent, nous le savons, systématiquement à notre attention et à notre analyse chaque fois que nous pensons les avoir compris. Nous pourrions dire que la langue est la partie rationnelle de notre système communicatif, le messager verbal entre l'inconscient et la conscience, entre notre intérieur et le monde extérieur, entre l'individu et la société. Il me semble donc évident, si nous voulons mieux nous comprendre nous-mêmes et saisir notre fonctionnalité en tant qu'être individuel et social, d'analyser la langue et ses capacités le plus soigneusement possible. La première étape est la grammaire, sans laquelle rien ne peut être décrit et qui comprend la phonétique, la morphologie, la formation des mots et la syntaxe. Cette analyse n'est d'ailleurs jamais seulement une fin en soi; elle sert toujours à mieux comprendre le fonctionnement entier de la langue. Et elle peut aboutir à des domaines encore plus élevés, notamment la stylistique, la rhétorique, l'étude de la conversation, de la narration, de la poésie.

Ce n'est pourtant que dans une perspective diachronique, c'està-dire historique, que l'on peut trouver des réponses à la question du

2. S. Pinker, The Language Instinct, passim. 
philosophe. Il est vrai que la compréhension de cet aspect, comme en littérature et en archéologie, ne va pas de soi. Mais les premiers pas ne sont guère difficiles, puisqu'ils n'exigent qu'une compétence légèrement supérieure au minimum linguistique naturel dans une langue moderne. Le surplus nécessaire peut résider dans la connaissance d'autres langues contemporaines, connaissance très répandue dans notre population, ou, ce qui est moins répandu, dans la connaissance de sa propre langue, ou d'autres langues, dans le passé.

Commençons avec un exemple qui nous montrera déjà à quel degré le passé est présent même dans les domaines les plus modestes de notre langue. Voici trois phrases de caractère simple et quotidien, mais dans cinq langues différentes:

$\begin{array}{lll}\text { manger un morceau de pain, } & \text { boire un verre de lait, } & \text { aller au lit } \\ \text { mangiare un pezzo di pane, } & \text { bere un bicchiere di latte, } & \text { andar a letto } \\ \text { comer un trozo de pan, } & \text { beber un vaso de leche, ir(se) al lecho / a la cama } \\ \text { ein Stück Brot essen, } & \text { ein Glas Milch trinken, zu Bett gehen } \\ \text { to eat a piece of bread, } & \text { to drink a glass of milk, to go to bed }\end{array}$

Lorsqu'on regarde un tel tableau, d'une structure bidimensionnelle, on commence presque automatiquement à faire des comparaisons: les mots pain - pane - pan, boire - bere - beber, lait - latte - leche et lit letto - lecho se ressemblent d'une manière frappante, et, pareillement, bread - Brot, drink - trinken, glass - Glas, milk - Milch, bed - Bett. Et l'on se demande aussitôt pourquoi, dans l'enseignement de l'allemand ou de l'anglais, les enseignants ne parlent presque jamais de tels parallèles. En apprenant l'italien ou l'espagnol, les francophones en sont sans doute plus conscients, parce que dans ce cas le rapport existe avec leur langue maternelle, qu'ils connaissent bien; mais encore une fois sans que l'on en parle directement. Il me semble parfois que l'attitude vis-àvis des langues étrangères consiste surtout à leur reprocher de contenir des mots différents de ceux dont on a l'habitude. Il est évident que la proximité linguistique entre les langues romanes, si l'une d'elles est notre langue maternelle, facilite l'apprentissage des autres. De même pour les germanophones, apprendre l'anglais est facilité par les rapports étroits entre les deux langues; mais il ne faut pas croire que nos professeurs d'anglais nous disent pourquoi les verbes comme sing-sang-sung, bring- 
brought-brought ressemblent tellement aux verbes allemands singen-sanggesungen, bringen-brachte-gebracht! Et voilà de nouveau notre question du philosophe "pourquoi?»!

Ce ne sont pourtant pas seulement les ressemblances qu'il faut observer, les différences sont aussi fort intéressantes et instructives, et elles sont d'ailleurs beaucoup plus difficiles à expliquer. Prenons l'expression anglaise a piece of bread. Il est évident que piece ressemble au français pièce et à l'italien pezzo, mais un francophone ne dirait jamais une pièce de pain. Pourquoi? Et qu'en est-il des mots morceau et trozo? Expliquer de telles différences n'est pas facile. L'espagnol vaso, au moyen duquel on boit du lait dans les pays hispanophones (ou plutôt du vino tinto, c'est-à-dire du "vin teint») nous pose moins de difficultés quoique nous n'utilisions normalement pas de vase pour boire. Mais que penser du verbe espagnol comer pour "manger"? Pour expliquer cette différence éclatante, les éléments nous manquent au premier regard.

C'est alors la comparaison des langues qui nous amène à poser la question du philosophe; et la question finale au sujet des langues humaines sera donc: pourquoi l'humanité utilise-t-elle des langues différentes et non pas une seule (ce qui rendrait notre vie, et notamment celle des fonctionnaires de l'Union Européenne à Bruxelles, beaucoup plus facile) et, s'il est naturel d'avoir une diversité de langues, comment les différences se font-elles, pourquoi existe-t-il malgré tout de multiples ressemblances et pourquoi, dans le détail, y a-t-il une ressemblance ici, mais une différence là?

Or, quel est le lien existant avec la tradition classique dont nous traitons ici? Il est impressionnant! Parmi les mots de notre exemple qui sont réguliers dans les trois langues romanes, au moins quatre sont très semblables en latin: pānis «le pain», lac (ou lacte) «le lait», lectus «le lit» et bibere "boire"; et nous observons que la version italienne en est normalement la plus proche. De plus, nous trouvons en latin manducāre "mâcher" qu'on met en rapport avec manger et mangiare, puis uitrum "le verre" et uās «récipient». Même la solution pour les verbes espagnols comer et ir nous y est offerte: ìre est le verbe latin normal pour «aller", tandis que les verbes correspondants à aller et andare ont en latin des significations plus particulières. Nous nous souvenons d'ailleurs qu'en français aussi ìre est encore présent, à savoir au futur et au conditionnel, tu iras et tu irais. Le cas de comer est semblable: le verbe latin manducāre a une signification particulière, et, là encore les Espagnols préfèrent 
utiliser le verbe qui est normal en latin. Dans cette dernière langue, ce verbe est comedere ou comesse, et il est utilisé, par exemple, dans le deuxième vers de la fameuse fable du corbeau et du renard que nous raconte d'une manière magistrale le poète Phèdre, latin Phaedrus, qui vivait à l'époque augustéenne:

\section{Cum dè fenestrā coruus raptum cäseum comesse uellet, celsā residens arbore...}

Alors que "Maître Corbeau, sur un arbre perché», voulait manger le fromage qu'il avait volé sur une fenêtre... ${ }^{3}$

Même ceux qui n'ont jamais fait de latin comprennent facilement presque tous ces mots, fenestra, coruus, raptus, cāseus, celsus, residère et arbor, qui ressemblent aux formes modernes fenêtre - finestra, corbeau corvo - cuervo, rapt - rápido - rape, caséine - queso - cheese, excellent Excelsior, résidence - residenza - residencia - residence, arbre - árbol - albero.

$\mathrm{Si}$, en montrant les rapports entre les mots romans et latins, j'ai évité jusqu'ici toute référence à la dimension historique, c'est intentionnellement que je l'ai fait! C'était ainsi que les savants - l'un des premiers à l'observer fut d'ailleurs Dante Alighieri - avaient d'abord décrit la parenté des langues romanes et du latin, et ce ne fut que beaucoup plus tard qu'on comprit que les langues romanes continuent le latin historiquement, qu'il y a donc une succession de langues ou d'étapes de langue de sorte que les langues romanes n'existaient pas encore quand le latin était vivant, et que le latin n'existait plus comme langue vernaculaire une fois les langues romanes nées. Mais il est aussi bien connu aujourd'hui que cela vaut pour les langues parlées et non nécessairement pour les langues écrites qui peuvent être utilisées, lues et écrites sans fin; toutefois, les parler une fois qu'elles sont «mortes» est chose artificielle et reste un but secondaire de l'enseignement.

Si nous adoptons maintenant une vision strictement historique, il faut insister sur un point important: les principes du changement linguistique, voire le fait même que les langues changent, furent établis à un moment plutôt récent dans l'histoire des sciences occidentales, à savoir autour de $1800^{4}$. On a alors découvert que les sons et les combinaisons

3. Phèdre, Fables 1.13.

4. G. Mounin, Histoire de la linguistique, p. 156-217. 
de sons dont les mots d'une langue sont composés changent avec le temps - très lentement, en fait, et rarement de manière perceptible pour un individu durant sa vie - et qu'au bout de quelques siècles, le système phonétique d'une langue peut être clairement différent de ce qu'il était au début. Prenons quelques exemples de tels changements phonétiques, illustrés par des paires de mots de structure phonétique semblable: le latin pänis et manus sont devenus pain et main, pane et mano, pan et mano; lacte «lait» et factum «fait» sont devenus lait et fait, latte et fatto, leche et hecho; nocte "(dans la) nuit" et octo "huit" sont devenus nuit et huit, notte et otto, noche et ocho.

Une origine phonétique identique a donc régulièrement abouti à un résultat identique dans chacune des langues, mais à des résultats différents d'une langue à l'autre. Le principe peut être décrit ainsi: le résultat d'un changement phonétique ne peut jamais être prévu, mais là où il y a un changement, il est le même pour tous les cas phonétiquement semblables. On s'imagine facilement quelle sensation fit cette découverte: elle ouvrit l'accès à la compréhension du comment et du pourquoi l'humanité connaît différentes langues!

Or, la ressemblance entre les langues romanes est tellement forte, même dans les détails de la conjugaison des verbes réguliers et irréguliers, qu'elle nous rend l'apprentissage mutuel très facile, et cela après un millénaire et demi de séparation suite à la disparition de l'Empire Romain d'Occident! La séparation était cependant beaucoup moins stricte qu'on pourrait le penser. Sauf le roumain, toutes les langues romanes sont entrées à nouveau en un contact étroit mutuel à l'époque carolingienne au plus tard. Et le latin en tant que langue d'église et d'érudition a empêché qu'elles s'éloignent trop les unes des autres; en fait, il a influencé l'histoire des langues européennes loin au-delà des frontières de la famille linguistique romane. Prenons l'anglais! D'une part, en 1066 les Normands ont importé le français en Grande-Bretagne, fait historique qui a énormément augmenté le vocabulaire de la langue anglaise. On a déjà vu l'exemple du mot pièce qui est devenu piece dans a piece of bread, mais il y a encore table, chair, plate, fork, cup et maints autres mots qui furent empruntés au français à ce moment-là. Ces emprunts furent même soumis à des changements phonétiques réguliers, typiques de l'anglais médiéval. Ainsi, reason et season correspondent à raison et saison, leurs équivalents actuels en français. En ce qui concerne feast "fête" et beast "bête», bien qu'ils aient maintenant un aspect tout à 
fait indigène, ressemblant à least et yeast d'origine germanique, l'emprunt d'un modèle français médiéval est évident, modèle qui d'ailleurs à cette époque-là était encore prononcé avec le $s$ original (voir en latin bestia, dies festus, etc.), dont aujourd'hui ne reste que le signe graphique de l'accent circonflexe. D'autre part, une partie encore plus grande du vocabulaire anglais, appelée les "hard words» et comprenant des mots souvent difficiles, longs et techniques, a été empruntée aux langues savantes latine et grecque dès le Moyen Age, parfois encore à travers le français, tels hydrodynamics, subservience, indemnification, puerperal, counterespionage, investigative. Bien que nous ne sachions souvent pas comment prononcer ces «hard words» en anglais, nous les comprenons normalement sans difficulté, beaucoup mieux de toute façon que la plupart des «soft words", à savoir les mots mono- ou dissyllabiques presque toujours d'origine germanique et d'un style quotidien, tels pat, pet, pit, pot, put, pate, peat, pout, daft, dale, dam, dank, darn, daub, daw, daze. Mais pour les gens de langue maternelle anglaise, la situation est inverse, d'où les épithètes «hard» et «soft». Du point de vue de la linguistique historique, les «soft words" n'ont d'ailleurs pas moins d'intérêt que les "hard words", puisqu'ils peuvent être comparés avec des mots apparentés dans d'autres langues germaniques. Il ne s'agit alors presque jamais d'emprunts mais de mots hérités d'une phase commune germanique avant l'émigration des Anglo-Saxons du continent au $\mathrm{V}^{\mathrm{e}}$ siècle apr. J.-C. Et là aussi, il vaut la peine d'observer étroitement les changements phonétiques réguliers. Ainsi on retrouve la différence dans la première consonne de drink et trinken, dans drive et treiben ou dead et tot; et celle du vocalisme dans dead et tot se retrouve dans bread et Brot, red et rot, lead "plomb" et Lot "aplomb" et même dans shred "déchirer, dépecer» et Schrot "menuise, petit plomb de chasse», ce qui ouvre la voie à la compréhension d'un grand nombre de rapports étymologiques fascinants.

Les langues germaniques, elles aussi, ont beaucoup emprunté au latin. La couche la plus ancienne de tels emprunts remonte à l'Empire Romain et comprend des mots de la vie quotidienne, tels Käse déjà mentionné, mais aussi Strasse, Platz, Küche, Wein, Büchse, Birne, Pfund, etc., et, de plus, une grande partie de la terminologie de l'église comme Kirche, Kloster, Bischof, Priester, Mönch, Messe, Opfer, Pfingsten, predigen. Une partie importante de ce vocabulaire est d'ailleurs en fin de compte d'origine grecque, à savoir Platz, Büchse, Kirche, Bischof, Priester, Mönch, Pfingsten. 
On retrouve les mots de cette couche ancienne dans presque toutes les langues de l'Europe occidentale, et, du fait qu'ils ont eu suffisamment de temps pour s'adapter phonétiquement aux langues qui les ont accueillis, on les reconnaît à peine comme des emprunts. Cela se voit surtout en anglais: cheese, street, place, kitchen, wine, box, pear, pound; church, cloister, bishop, priest, monk, mass, offer, Pentecost, preach. On suppose d'ailleurs que les Anglo-Saxons avaient appris les plus anciens d'entre eux déjà avant leur émigration de l'Allemagne; d'autres n'ont atteint l'Angleterre qu'avec les Normands après 1066, notamment place, cloister, to preach. Même les langues slaves occidentales présentent une partie de ces emprunts, qu'elles ont acquis lors de la christianisation dès 800 apr. J.-C. environ; par exemple le polonais, où l'on trouve plac, kuchnia, wino, puszka, funt; cerkiew (orthod.) ${ }^{5}$, klasztor, biskup, mnich, msza, ofiara, dont quelques-uns présentent la forme allemande, notamment plac, prononcé [plats], et funt.

Le phénomène des "hard words" n'est pas réservé à l'anglais. Dès la Renaissance, le vocabulaire de toutes les langues européennes a été enrichi de milliers de mots savants latins et grecs. Et il est évident que les locuteurs de langues romanes y ont eu un énorme avantage, puisqu'ils connaissaient les racines d'une grande partie de ces mots, surtout ceux d'origine latine dont les racines étaient déjà présentes dans leur vocabulaire de base. Mais puisque ces derniers mots, hérités directement du latin, avaient entre-temps été modifiés par des changements phonétiques (notamment en français où ces changements étaient particulièrement forts et fréquents), on observe maintenant tout une série de paires étymologiques, où un mot hérité survit à côté de son "clone» emprunté secondairement au latin savant: orteil et article, aôेt et auguste, écouter et ausculter, chance et cadence, forge et fabrique, frêle et fragile, métier et ministère, raison et ration, sanglier et singulier, étroit et strict.

Dès le Moyen Age, où elles étaient entrées en un contact très intensif qui perdura jusqu'à nos jours de paneuropéanisme, les langues "modernes» ont aussi emprunté des mots - et non seulement des mots, mais aussi d'autres traits linguistiques, notamment syntaxiques - les unes des autres. Très souvent il est même impossible de dire quelle langue fut le modèle de quelle autre pour tel ou tel emprunt. On a déjà vu qu'après 1066, l'anglais importa une grande quantité de mots français. Déjà auparavant, il

5. Le mot courant pour "église» est kościót, du latin castellum. 
avait intégré des mots "vikings", ce qui a, entre autres, produit des clones germaniques amusants comme shirt (anglais originel) et skirt (emprunt nordique). La littérature médiévale paneuropéenne avec ses nombreuses traductions et imitations ("Chanson de Roland", "Roman de Troie", "Arthur» et ses "Chevaliers de la Table ronde», notamment "Perceval", etc.), les savants migrant entre les nouvelles universités (Bologne, Paris, Oxford, Prague, etc.), les mariages internationaux entre les maisons princières de toute l'Europe et les sciences qui commençaient d'apparaittre aux $\mathrm{XVI}^{\mathrm{e}}$ et XVII $\mathrm{X}^{\mathrm{e}}$ siècles devaient encore renforcer les contacts linguistiques et le besoin d'exprimer tout ce que pouvaient exprimer les autres langues vivantes et, surtout, le latin. Même le vocabulaire de tous les jours était fortement standardisé, notamment par le commerce européen et mondial. Ainsi, on observe, par exemple en allemand, des centaines d'emprunts du français: Vase, Vitrine, exzellent, originell, Residenz, Platte, Saison et Staatsraison. Plusieurs mots ont fait un détour via l'anglais, comme Leasing (vis-à-vis de Laisser-faire), Couch (prononcé [kaut J], vis-à-vis de kuschen et kuscheln) et Europa Cup (vis-à-vis de Coupe Dänemark). Et tout cela, et beaucoup plus, vient finalement du latin ou du grec.

La curiosité du linguiste ne s'arrête pas encore. La question se pose également de savoir si la ressemblance entre l'allemand essen et le latin (com)esse, entre Nacht et noctem, acht et octō, entre ist et est, sind et sunt, et encore entre sechs - sieben - neun - zehn et sex - septem - novem - decem s'explique, elle aussi, par un emprunt du latin il y a 2000 ans. Mais dans ce cas, la réponse doit être clairement négative, car ces mots et leur histoire nous emmènent dans un passé encore plus lointain. En effet, le latin et les langues germaniques sont apparentées elles aussi. Elles appartiennent à la famille linguistique indo-européenne, qui présuppose l'existence d'une langue commune autour de 3000 ou 4000 av. J.-C. Font aussi partie de cette grande famille les langues slaves, baltes, celtiques, le grec, même le sanskrit en Inde et beaucoup d'autres. C'est précisément avec la découverte de cette grande famille de langues qu'a été fondée, au début du XIX ${ }^{\mathrm{e}}$ siècle, la grammaire comparée des langues indoeuropéennes, plus précisément avec le premier livre entièrement dédié à la comparaison de ces langues et surtout de la morphologie de leur système verbal, publié en 1816 et dont l'auteur était Franz Bopp ${ }^{6}$. La découverte

6. F. Bopp, Über das Conjugationssystem der Sanskritsprache in Vergleichung mit jenem der griechischen, lateinischen, persischen und germanischen Sprache. 
de Bopp a tellement enthousiasmé le monde savant de l'époque que le puissant ministre de la jeune Prusse, Wilhelm von Humboldt, créa pour Bopp dans sa nouvelle Université de Berlin la toute première chaire de linguistique et de langues orientales du monde. Dès lors, la discipline de la linguistique historique et comparative fit d'énormes progrès dans la description et la compréhension des changements linguistiques à tous les niveaux de la langue. La parenté des langues et des dialectes, leur développement divergent dans des périodes de ségrégation et de séparation, et, inversement, leur développement convergent lors d'une réunion de groupes de locuteurs auparavant séparés, sont maintenant des concepts bien établis dans la science du langage.

L'un des plus importants phénomènes de l'histoire comparative du vocabulaire de nos langues fournira le dernier point de notre plaidoyer en faveur d'une Europe des langues vue dans une perspective historique. En apprenant l'allemand, les francophones se demandent sans doute régulièrement pourquoi les correspondances lexicales littéraires, religieuses ou techniques sont si souvent sémantiquement proches, mais formellement différentes de ce qu'on utilise dans les langues romanes et même en anglais. Pourquoi en allemand pour le nominatif, génitif, datif, etc., on utilise normalement le mot der Fall au lieu de Kasus, correspondant à le cas, the case? Pourquoi grossmütig ou grossherzig pour magnanime, magnanimous? Pourquoi der Heilige Geist pour le Saint-Esprit, the Holy Spirit? Pourquoi die Begeisterung pour l'inspiration, the inspiration, où nous retrouvons le rapport entre Geist et esprit - spirit de l'exemple précédent? En linguistique, le phénomène auquel nous avons affaire ici s'appelle "calque». Un calque est un mot emprunté qui n'est pas adopté tel quel, mais auquel on a fait, pour ainsi dire, changer de vêtements en créant dans la langue qui l'emprunte un nouveau mot (calque morphologique) ou simplement en imposant le sens emprunté à un mot préexistant avec un sens voisin (calque sémantique). Il s'agit là d'un phénomène extrêmement fréquent, surtout dans les langues septentrionales européennes, à savoir les langues celtiques, le germanique, le slave, le balte, mais aussi le hongrois, le finnois et l'estonien, qui font aussi partie de la culture de l'Europe occidentale tout en n'appartenant pas à la famille linguistique indo-européenne. Il est donc possible qu'un mot ait un aspect allemand, ou polonais, ou finnois, mais qu'on y trouve, en «fouillant", un concept tout-à-fait classique en arrière-fond, directement pris au latin: cāsus, magnanimus, spiritus et inspìrātiō. L'anglais connaît d'ailleurs aussi 
ce procédé, mais il adopte tout aussi volontiers la méthode de l'emprunt direct. Ainsi, magnanimous coexiste avec great-hearted, the Holy Spirit avec the Holy Ghost, mais pour les emplois du latin casus l'anglais n'utilise que case, que ce soit au sens grammatical ou dans un sens plus large, par exemple dans ce cas-in this case (all. in diesem Fall), au cas où-in case (all. falls; für den Fall, dass), c'est le cas-it is the case (all. es ist der Fall). L'ancien mot germanique the fall, pour sa part, est strictement réservé aux sens concrets, à savoir "chute, cascade, pente, automne, etc.»; il n'a pas été adopté, comme ce fut le cas de son cousin allemand, pour des calques sémantiques motivés par le latin.

Or notre histoire linguistique de la tradition classique n'est pas encore complète avec une démonstration de l'influence multiple du latin sur les langues de l'Europe actuelle. En fait, j'ai choisi ces trois exemples de calques linguistiques cāsus, magnanimus, spìritus - inspìrātiō parce que les mots français ou latins concernés ainsi que leurs significations ne sont pas des inventions romaines. Ils remontent en effet au grec, et les Romains ont agi de la même manière que les Allemands beaucoup plus tard: ils ont traduit, calqué les modèles grecs. Le mot cāsus, déjà utilisé par Cicéron et Varron pour désigner les cas grammaticaux, est un calque du grec $\pi \tau \tilde{\omega} \sigma \iota \varsigma$, dérivé du verbe cadere, «tomber », comme le subs-

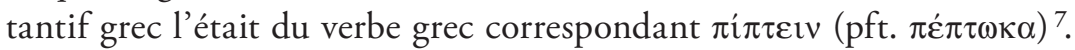
Ladjectif magnanimus de son côté est un calque de $\mu \varepsilon \gamma \alpha \dot{\theta} \theta \nu \mu \varsigma$, épithète de divinités et d'animaux déjà présent chez Homère. Le troisième cas, celui de spiritus et inspiratiō, quant à lui, est tellement fascinant que nous allons le développer un peu plus en détail, puisque son histoire semble ne pas avoir été suffisamment bien comprise et décrite jusqu'ici.

Le latin inspirrāre signifie littéralement "remplir quelqu'un de souffle (divin)». La composition in + spīrāre "respirer» avec un sens factitif et transitif est attestée pour la première fois chez Virgile ${ }^{8}$. Il n'y a aucun doute qu'il s'agit ici d'un calque (sémantique et syntaxique) d'après le

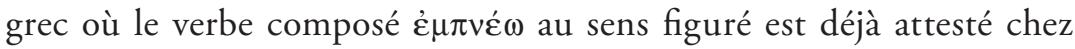

7. La raison pour laquelle les formes de la déclinaison reçurent cette désignation en grec n'est d'ailleurs pas claire.

8. Le verbe semble avoir été créé par Lucrèce avec le sens intransitif «respirer» (cf. De la nature 4.1109). Virgile a d'abord ajouté l'usage transitif dans les Géorgiques (4.236 sq.), puis, dans l'Enéide, le sens de l'inspiration divine, érotique (1.687-688, Vénus à Cupidon) et poétique-prophétique (6.11-12, Sibylla). 


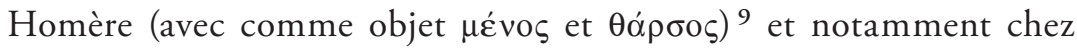
Hésiode (avec comme objet $\alpha$ v̉ò̀ $\theta \dot{\varepsilon} \sigma \pi \mathrm{\iota})^{10}{ }^{10}$, tandis que pour le substantif et l'adjectif la composition avec غ̇ $\pi \dot{i}$ est plus courante: le substantif

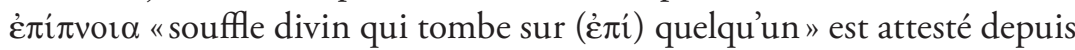
Eschyle ${ }^{11}$, et l'adjectif غ̇ $\pi \dot{i} \pi v o o \zeta$, de structure possessive ("ayant $\pi v o \eta ́$ sur soi-même») mais réinterprété avec un sens passif "pris par le souffle divin", est fréquent notamment chez Platon ${ }^{12}$. Plus tard, la correspondance des racines $\pi v \varepsilon(F)$ - et spirr-, établie par ce calque classique, a été réutilisée dans le Nouveau Testament latin pour le calque de Spïritus

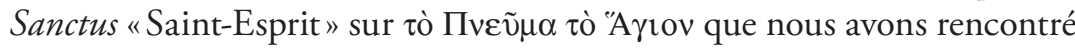
plus haut et qui, de son côté, est fortement influencé par la tradition judaïque. Or, pour les Grecs et les Romains, le $\pi v \varepsilon \tilde{v} \mu \alpha$ et le spiritus de ces images religieuses était normalement plus qu'un simple «souffle», et, en fait, les Grecs - et seulement eux - avaient une expression qui signalait un concept beaucoup plus puissant, à savoir év $\theta \varepsilon o \varsigma$, de nouveau un composé possessif de formation très archaïque «ayant la divinité en soi-

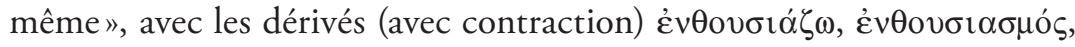

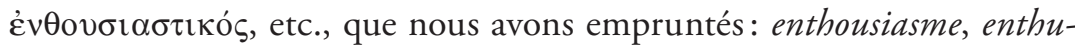
siasm, Enthusiasmus, etc. Cette famille de mots, à la différence de celle de $\dot{\varepsilon} \pi \dot{i}+\pi v \varepsilon(F)-/ \pi v o(F)-$, n'a pas uniquement une signification positive, mais, selon la divinité et la situation de son activité, elle peut être positive ou négative, bienfaisante ou menaçante. Cette ambiguïté du divin, on le sait, a eu une très grande importance pour le caractère de la religion des Grecs anciens, si on se rappelle d'un côté l'inspiration divine des poètes et musiciens et de l'autre celle des Bacchantes chez Euripide, ou celle d'Eros, divinité dont l'ambiguïté est exemplaire. Les Romains, pour leur part, n'ont pas eu la possibilité de former des calques précis et élégants sur ěv $\theta \varepsilon \circ \varsigma$ et ses dérivés: ${ }^{*}$ indeus/*indivvus étaient impossibles non seulement à cause de l'ambiguïté du latin $i n^{-13}$ mais aussi à cause de la perte préhistorique de la catégorie des composés possessifs dans cette langue, et, de même, un verbe *indeāre, *indìvāre aurait été incongru. Ils se contentaient donc du calque inspirrāre, mais le limitaient en même

9. Homère, Iliade 20.110, Odyssée 9.381.

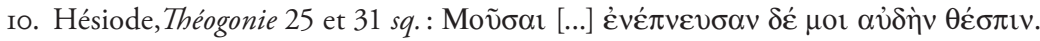

"(les) Muses [...] m’inspirèrent des accents divins" (trad. Paul Mazon).

II. Eschyle, Les Suppliantes 17, 43, 577.

I2. Platon, Cratyle 428c, Ménon 99d, Le Banquet 181c.

13. Sont restés plus clairement distincts le grec év-vs $\dot{\alpha}$-, et l'allemand in-vs un-. 
temps à ses sens positifs. Le concept large d'év $\theta \varepsilon o \varsigma$, impossible à traduire dans une langue moderne européenne, le latin inclus, reste donc dans son sens universel un concept purement grec (il semble même qu'on puisse en tirer un principe linguistique important, à savoir que certains concepts et certaines idées sont liés à la faculté d'être exprimés d'une manière brève et précise par la langue en question). Déjà le latin a dû

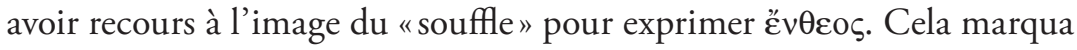
la fin du concept ambigu et de la double signification de l'«inspiration", et l'Europe devait dès lors se contenter - de nos jours encore - de deux termes, le terme positif de l'inspiration (all. Begeisterung) et le terme négatif de l'obsession (all. Besessenheit). En fait, même le mot enthousiasme directement emprunté a perdu complètement son côté négatif et menaçant, ce qui nous montre la distance entre la religion archaïque et classique des Grecs et celle des Romains, dont les influences grecques étaient surtout hellénistiques, et, encore plus, entre la religion des Grecs et le christianisme, qui depuis l'Antiquité a marqué de son empreinte la façon de penser et de parler en Occident.

En ce qui concerne l'origine de l'image du souffle divin, on peut se demander si elle ne viendrait peut-être pas du contexte précis de l'oracle de Delphes. De ce fameux endroit nous sont parvenues plusieurs descriptions qui nous apprennent que la Pythie puisait son inspiration de l'eau de la source Kassotis ${ }^{14}$ ou d'une vapeur sortant de la terre. Voici ce qu'en dit Strabon:

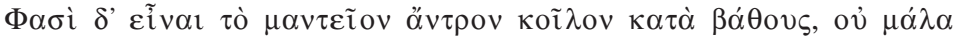

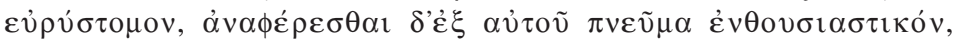

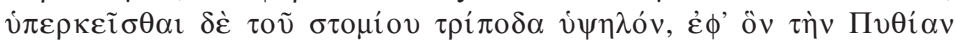

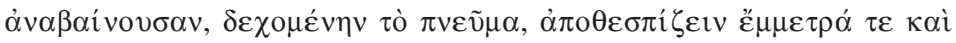

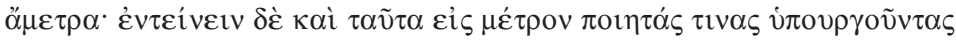
$\tau \tilde{\omega}$ i $\varepsilon \rho \tilde{\varphi}$.

On dit que l'oracle est une cavité qui pénètre profondément dans le sol, avec un orifice étroit d'où s'exhale une émanation propre à susciter un transport surnaturel. Sur l'orifice est installé un trépied élevé sur lequel monte la Pythie. Pénétrée du souffle inspirateur, elle rend des oracles en vers ou en prose. Ces derniers sont alors mis en vers par des poètes attachés au service du sanctuaire ${ }^{15}$.

I4. Pausanias, Description of Greece 10.24.7.

15. Strabon, Géographie 9.3.5. 
L'oracle avait été découvert, selon les Anciens, par des bergers dont les chèvres commençaient à sauter d'une manière étrange en s'approchant d'un certain endroit ${ }^{16}$. Plutarque, qui avait été prêtre à Delphes et connaissait donc mieux les lieux et les procédés que tout autre écrivain, nous en parle aussi ${ }^{17}$. Ces observations grecques ont récemment été confirmées d'une manière plausible par l'observation de deux failles géologiques qui se croisent très probablement sous le temple d'Apollon et par la mise en évidence de traces du gaz hallucinogène éthène ou éthylène $(\mathrm{C} 2 \mathrm{H} 4)$ et d'autres substances toxiques ${ }^{18}$. Or, le mythe de la découverte de cet effet hallucinogène à l'endroit du futur oracle nous entraîne dans un passé lointain et semi-mythique où les bergers et leurs chèvres occupaient encore le site. Il est vrai, la première référence directe d'un mot dérivé de la racine de $\pi v \varepsilon ́ \omega$ à la mantique apollinienne se trouve, sauf erreur de ma part, chez Platon ${ }^{19}$, mais on a vu que l'inspiration divine du poète par les Muses, dont Apollon était le conducteur, est déjà attestée chez Hésiode, et l'on sait que les oracles étaient proclamés en hexamètres. Il semble donc très probable que les vapeurs inspiratoires ont été découvertes à une époque très reculée; sans quoi le sanctuaire et le temple d'Apollon ne se trouveraient pas exactement à l'endroit du croisement des deux failles géologiques; et, par conséquent, l'image de l'inspiration par un souffle divin pourrait très bien avoir été "inspirée»

I6. Diodore, The Library of History 16.26; Pausanias, Description of Greece 10.5.7.

17. Plutarque, De Pythiae oraculis 17 (mor. 402B), où le fait que la Pythie a cessé de prophétiser en vers est expliqué par l'extinction du souffle : $\tau$ oṽ $\pi v \varepsilon v ́ \mu \alpha \tau o \varsigma \pi \alpha v \tau \alpha \dot{\pi} \pi \alpha \sigma v v$

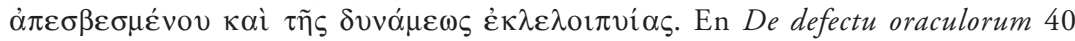

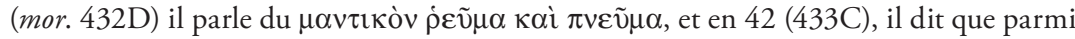
les exhalaisons ( $\dot{\rho} \varepsilon v ́ \mu \alpha \tau \alpha)$ que la terre fait jaillir, celles de Delphes sont les seules à disposer les âmes à l'enthousiasme et à la révélation de l'avenir. Ensuite, il parle d'un ber-

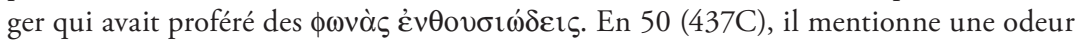
agréable et parfumée qui remplit le local où ceux qui consultent les dieux sont assis.

I8. J. Z. de Boer, J. R. Hale et J. P. Chanton, "New evidence for the geological origins of the ancient Delphic oracle (Greece)»; H. A. Spiller, J. R. Hale, J. Z. de Boer, "The Delphic oracle: a multidisciplinary defense of the gaseous vent theory"; J. R. Hale, J. Z. de Boer, J. P. Chanton, H. A. Spiller, "Questioning the Delphic oracle». Pour ces informations, je me base également sur le travail de maturité de Xenja Herren, Kommentar zu Pausanias: Textstellen 10.5.5-5.11; 10.6.5; 10.12.1-12.3 und 10.12.8-12.11 (Gymnase de Langenthal, novembre 2004), sous la direction de Catherine Trümpy et de moi-même.

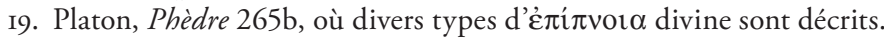


par des observations faites à Delphes même à l'époque homérique, voire pré-homérique.

Pour conclure ces petites "fouilles de mots", nous n'hésitons pas à affirmer que dans la plus grande partie du vocabulaire culturel de nos langues, et même dans bien des mots de tous les jours, se cachent plus ou moins ouvertement l'influence immense de l'Antiquité, latine et souvent même grecque, surtout si l'on n'oublie pas de prendre en compte les calques. De plus, un très grand nombre de mots de nos langues actuelles sont encore plus anciens, beaucoup plus anciens, et se perdent dans l'ombre du passé, indo-européen ou même plus lointain. Ainsi, cet outil quotidien qu'est notre langue se révèle être un trésor immensément riche en tradition, tradition classique surtout, au moins dans le vocabulaire de la littérature, de l'art et de la science.

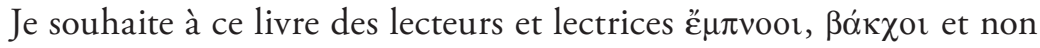

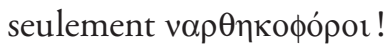

Rudolf WACHTER

Universités de Lausanne et de Bâle 


\section{BIBLIOGRAPHIE}

\section{Textes}

Diodore, The Library of History 7, Books XV, 20 - XVI, 65, with an English translation by Charles L. Sherman, The Loeb Classical Library 389, Cambridge (MA), Harvard UP, 1980.

Eschyle, Tragédies, Tome I: Les Suppliantes - Les Perses - Les Sept contre Thèbes - Prométhée enchaîné, texte établi et traduit par Paul Mazon, Paris, Les Belles Lettres, 2002.

Hésiode, Théogonie - Les Travaux et le Jours - Bouclier, texte établi et traduit par Paul Mazon, Paris, Les Belles Lettres, 2002.

Homère, Iliade, tome IV, texte établi et traduit par Paul Mazon, Paris, Les Belles Lettres, 2002.

—, L'Odyssée, tome II, texte établi et traduit par Victor Bérard, Paris, Les Belles Lettres, 2002.

Lucrèce, De la nature, texte établi et traduit par Alfred Ernout, 2 vols, Paris, Les Belles Lettres, 1985-2002.

Pausanias, Description of Greece 4, Books VIII (XXII) - X, with an English translation by William Henry Samuel Jones, The Loeb Classical Library 297, Cambridge (MA), Harvard UP, 1979.

PhÈDre, Fables, texte établi et traduit par Alice Brenot, Paris, Les Belles Lettres, 1989.

Platon, Euvres complètes, Tome III, $2^{e}$ partie: Gorgias - Ménon, texte établi et traduit par Alfred Croiset, Paris, Les Belles Lettres, 2003.

—, Euvres complètes, Tome IV, $2^{e}$ partie: Le Banquet, texte établi et traduit par Paul Vicaire, Paris, Les Belles Lettres, 2002.

—, Euvres complètes, Tome IV, $3^{e}$ partie: Phèdre, texte établi par Claude Moreschini et traduit par Paul Vicaire, Paris, Les Belles Lettres, 2002.

—, Euvres complètes, Tome IV, $1^{\text {re }}$ partie: Phédon, texte établi et traduit par Paul Vicaire, Paris, Les Belles Lettres, 2002. 
—, Euvres complètes, Tome V, $2^{e}$ partie: Cratyle, texte établi et traduit par Louis Méridier, Paris, Les Belles Lettres, 2003.

Plutarque, Euvres morales, tome VI: Dialogues pythiques, texte établi et traduit par Robert Flacelière, Paris, Les Belles Lettres, 1974.

Strabon, Géographie, tome VI (Livre IX), texte établi et traduit par Raoul Baladié, Paris, Les Belles Lettres, 1996.

Virgile, Enéide, texte établi et traduit par Jacques Perret, 3 vols, Paris, Les Belles Lettres, 1978-1980-2002.

—, Géorgiques, texte établi et traduit par Eugène de Saint-Denis, Paris, Les Belles Lettres, 1995.

\section{Etudes}

Boer, Jelle Zeilinga de, Hale, John R. et Chanton, Jeffrey P., «New evidence for the geological origins of the ancient Delphic oracle (Greece)", Geology, 29/8 (August 2001), p. 707-710.

Bopp, Franz, Über das Conjugationssystem der Sanskritsprache in Vergleichung mit jenem der griechischen, lateinischen, persischen und germanischen Sprache, Frankfurt am Main, 1816.

Hale, John R., Boer, Jelle Zeilinga de, Chanton, Jeffrey P. et Spiller, Henry A., "Questioning the Delphic oracle», Scientific American, 289/2 (2003), p. 66-73.

Herren, Xenja, Kommentar zu Pausanias: Textstellen 10.5.5-5.11, 10.6.5, 10.12.1-12.3 und 10.12.8-12.11, sous la direction de Catherine Trümpy et Rudolf Wachter, Gymnase de Langenthal, novembre 2004, inédit.

Mounin, Georges, Histoire de la linguistique. Des origines au XXe siècle, Paris, Quadrige, 1985.

Pinker, Stephen, The Language Instinct, New York, William Morrow, 1994.

Spiller, Henry A., Hale, John R. et Boer, Jelle Zeilinga de, "The Delphic oracle: a multidisciplinary defense of the gaseous vent theory", Journal of Toxicology and Clinical Toxicology, 40/2 (2002), p. 189-196. 